\title{
Parámetros de los reparos anatómicos del agujero del nervio mentoniano para cirugía oral
}

\author{
Parameters of the anatomical marks of the mental nerve hole for oral \\ surgery
}

\begin{abstract}
Resumen
El Nervio Mentoniano (NM) constituye una de las estructuras anatómicas importantes a identificar preservar y evitar lesionarlo. El conocimiento de la disposición y las variantes anatómicas del NM es imperativo para la ejecución de procedimientos quirúrgicos que involucren el área mentoniana. Objetivo: El propósito de este estudio fue determinar mediante la observación en preparados anatómicos de cadáveres humanos la frecuencia de la ubicación y emergencia de agujero del NM, sus accesorios, presencia de bucles y sus relaciones con el reborde alveolar y el borde mandibular. Material: Se disecaron 31 hemimandíbulas de cadáveres. Resultados: se encontró que la distancia más frecuente entre la emergencia del NM con respecto al reborde alveolar y al reborde mandibular fue del rango de 11 a 15.5 $\mathrm{mm}$ en un $57.6 \%$ y de 11 a $15.5 \mathrm{~mm}$ en el $72.7 \%$ respectivamente. La relación del NM con respecto a los dientes fue que el $63.6 \%$ está a la altura de la 2a PMI. El 18.2\% presentó nervio mentoniano accesorio y la ubicación más frecuente fue superior al NM en un 66.7\%; no se encontró la presencia de bucles. Conclusión: La emergencia del agujero del nervio mentoniano en relación a las piezas dentarias de personas en el Perú, se da mayormente por debajo de la 2a PMI en un 63,6\%, que coincide con la mayoría de investigaciones, la presencia de NM accesorios se halló en un $18 \%$, porcentaje que es mayor en comparación con otros estudios, por lo que se recomienda un mayor cuidado en las cirugías en la región mentoniana.
\end{abstract}

Palabras clave: Anatomía; mentón; mandíbula; sistema nervioso.

\begin{abstract}
The mental nerve $(\mathrm{MN})$ is one of the anatomical structures important to identify, preserve and avoid injure. The knowledge of the layout and anatomical variants of $\mathrm{MN}$ is imperative for the performance of surgical procedures involving the chin area. Objective: The purpose of this study was to determine, the location and frequency of emergency hole of $\mathrm{MN}$, accessories nerves, presence of loops and their relations with the alveolar ridge and the mandibular border, by observing prepared anatomical human corpses. Methodology: 31 mandibles of corpses were dissected. Results: It was found that the most frequent distance between the emergency $\mathrm{MN}$ with respect to the mandibular alveolar ridge was the range of 11 to $15.5 \mathrm{~mm}$ at $57.6 \%$ and 11 to $15.5 \mathrm{~mm}$ in $72.7 \%$ respectively. NM's relationship with respect to the teeth was that $63.6 \%$ of the cases is at the same height of the PMI $2 \mathrm{a}, 18.2 \%$ had accessory mental nerve and the most frequent location was upper than $\mathrm{MN}$ in $66.7 \%$ of the cases; the presence of loops was not found. Conclusion: the MN hole emergency occurs mostly below the PMI 2a in 63.6\% of peruvian people, which coincides with the majority of investigations. The presence of accessories MN was found in $18 \%$, which is higher compared to other studies, so greater care in surgeries in the chin area is recommended.
\end{abstract}

Keywords: Anatomy; chin; jaw; nervous system.

\section{Introducción}

El agujero mentoniano es un reparo anatómico que se define como una apertura en la superficie bucal de la mandíbula, dicha estructura proviene del extremo anterior del conducto dentario inferior. ${ }^{1-7}$

Es importante conocer las diferentes posiciones del agujero mentoniano, para evitar riesgos de lesión del NM
Artículo Original

\author{
Juana Delgadillo-Ávila1, \\ Carlos Campodónico-Reátegui², \\ Sergio Alvarado-Menacho ${ }^{3}$, \\ María Castañeda-Mosto ${ }^{4}$, \\ Sofía Espinoza-Escajadillo", \\ Luis Maita-Veliz ${ }^{5}$, \\ Sixto Grados Pomarino ${ }^{5}$, \\ Ada Delgado-Yauyo ${ }^{6}$, \\ Vladimir Huayta-Alarcón ${ }^{6}$
}

1. Departamento Académico de Ciencias Básicas

2. Departamento Académico de Estomatología Preventiva y Social

3. Departamento Académico de Estomatología Rehabilitadora

4. Departamento Académico de Estomatología Pediátrica

5. Departamento Académico Médico Quirúrgico 1-5. Facultad de Odontología de la Universidad Nacional Mayor de San Marcos, Perú.

6. Facultad de Odontología de la Universidad San Martin de Porres, Perú.

Correspondencia

Dra. Juana Rosa Delgadillo Ávila

Calle Ramon Cerdeira 175- San Borja.

Lima, Perú.

Correo electrónico: juanadelgadillo@yahoo.com

Coautores

Campodónico: ccampo_04umber@hotmail.com Alvarado: salvarado4@hotmail.com Espinoza: sofiabelinda@hotmail.com Maita: luisvimv21@hotmail.com Grados: sixtogp@gmail.com

Delgado: adita_delgado@hotmail.com

Huayta: vladimirhalex@gmail.com

Recibido: 16-05-15

Aceptado: 10-07-15 en los procedimientos anestésicos, intervenciones quirúrgicas, en la colocación de implantes y otros procedimientos invasivos, en los cuales podrían crearse complicaciones como la parálisis del nervio mentoniano, hemorragias, etc. ${ }^{1,2,8,9,10-21}$ (Fig 1)

El NM constituye una de las estructuras anatómicas más importantes para identificar y así preservar y evitar lesionarlo.
El conocimiento de la disposición y las variantes anatómicas del NM es importante para la ejecución de procedimientos que involucren el área mentoniana, en los aspectos morfológicos, estéticos y funcionales ${ }^{22-32}$.

Según las investigaciones, la ubicación del agujero mentoniano en su mayoría coinciden que está a nivel de la segunda premolar inferior, como los estudios 
realizados por Kim et, al. Corea $2006^{4}$; Amorim et. al. Brasil 2008 22 ; Liyian $2009^{14}$; Llayperuma 2009 ${ }^{23}$; Agarwal y Gupta Surat 201120; Mbajiorgu, Mawera y Zivanovic Zimbabwe $1998^{12}$ quienes encontraron que la ubicación estaba por debajo del $2^{\text {do }}$ PMI en el lado derecho y posterior a ella en el lado izquierdo.

De acuerdo con Shaik et, al. India-2012 21 , también realizaron un estudio en 70 mandíbulas, donde encontraron que la ubicación del foramen mental fue hallado $72,43 \%$ en línea con el eje longitudinal de los $2^{\text {os }}$ PMIs y $13,57 \%$ estaban presentes entre la $1^{\text {ra }}$ PMI y la $2^{\text {da }}$ PMI.

Con respecto a la distancia del agujero mentoniano al reborde alveolar y el borde mandibular, Agarwal y Gupta
Surat $2011^{20}$, en su estudio con mandíbulas humanas disecadas en Gujarat, encontraron que las mediciones respectivas desde el borde superior del agujero a la cresta alveolar fue de 14.05 $\mathrm{mm} \pm 3.05 \mathrm{~mm}$ en el lado derecho y $13.82 \mathrm{~mm} \pm 3.06 \mathrm{~mm}$ en el lado izquierdo.

Shaik et, al. India-2012 21 , encontraron que la distancia media entre el foramen mentoniano y el margen alveolar fue de $13,80+3,56 \mathrm{~mm}$ en el lado derecho y de $13,21+3,75 \mathrm{~mm}$ a la izquierda el estudio realizado por Kim et, al. Corea 20064, quienes evaluaron 112 forámenes mentales 72 en varones y 40 en mujeres que resultaron expuestos durante la operación, encontrando que la distancia media entre el borde superior del foramen mentoniano y la parte inferior de la mandíbula, fue de
$14,33 \mathrm{~mm}$ por medición directa y de $16,52 \mathrm{~mm}$ en mediciones radiográficas.

Singh y Srivastav India $2010^{\circ}$, encontraron la presencia de forámenes mentales accesorios en un $8 \%$ en el lado izquierdo y $5 \%$ en el lado derecho.

Así mismo Gupta y Soni India 2012 25 , encontraron que la presencia de forámenes accesorios se evidencio en 8 de cada 120 mandíbulas y su posición fue unilateral.

El propósito de este estudio fue determinar mediante la observación en preparados anatómicos de cadáveres humanos la frecuencia de la ubicación y emergencia del NM, nervios accesorios, presencia de bucles y sus relaciones con el reborde alveolar y el borde mandibular.

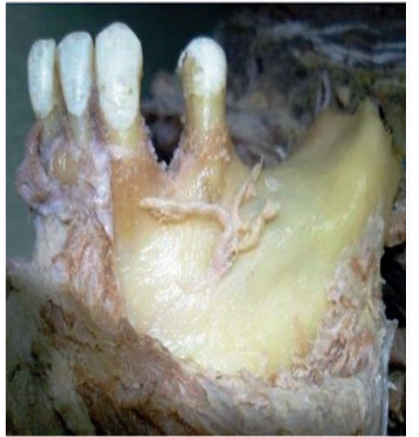

Fig. 1 Nervio mentoniano y reparos anatómicos

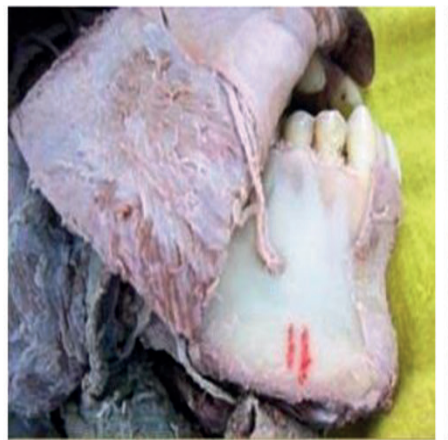

Fig. 2

Nervio mentoniano disecado

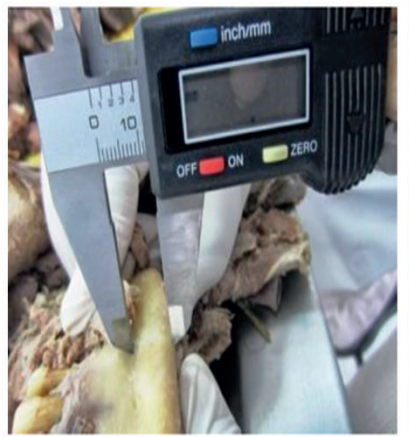

Fig. 3 Estudio antropométrico con calibrador digital

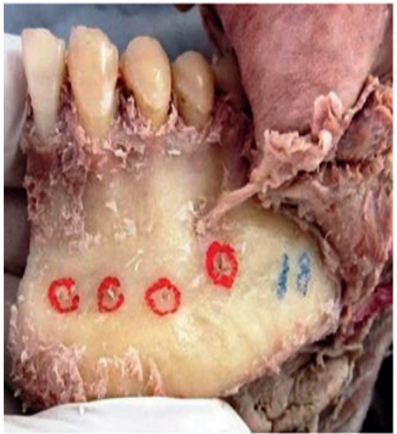

Fig. 4 Nervios mentoniaanos accesorios (18.2\%)

\section{Materiales y método}

El estudio fue descriptivo de corte transversal, se disecaron 30 hemimandíbulas dentadas y 3 hemimandíbulas edéntulas correspondientes a cadáveres de hombres adultos, de razas mestizas; ubicadas en el anfiteatro de la Facultad de Odontología de la Universidad Nacional Mayor de San Marcos en Lima, Perú.

Se clasificó y enumeró el material cadavérico. Se procedió a diseccionar la región mentoniana en las cabezas de los cadáveres, descubriendo en estas los reparos anatómicos del NM y sus relaciones con: las piezas dentarias, el reborde alveolar y mandibular y establecer la presencia de bucles y nervios accesorios (Fig. 2)

Se realizó el estudio antropométrico de los casos con mediciones cuantitativas con calibradores digitales y posterior análisis estadístico de los resultados, utilizando el programa SPSS versión 19. (Fig. 3) (b)ninguno.

\section{Resultados}

La emergencia del nervio mentoniano en relación al reborde alveolar fue de 11 a $15,5 \mathrm{~mm}(57,6 \%)$, seguido de 16 a $20 \mathrm{~mm}(27,3 \%)$. Donde la media fue 13,89mm, y la Ds. de 2,72. (Gráfico 1).

De igual forma la emergencia del nervio mentoniano en relación al reborde mandibular fue de 11 a 15,5 $\mathrm{mm}(72,7 \%)$, seguido de 16 a $20 \mathrm{~mm}$ $(21,2 \%)$, donde la media fue 14,69 mm y la Ds. de 1,88.

La emergencia del nervio mentoniano en relación al ápice de los dientes fue de $63,6 \%$ a la altura de la $2^{\text {da }}$ PMI, de $18,2 \%$ entre la $1^{\text {ra }}$ PMI y la $2^{\text {da }}$ PMI, de 9, $1 \%$ entre la $2^{\text {da }}$ PMI y la $1^{\text {ra }}$ Molar inferior, quedando un $9,1 \%$ de la muestra sin relacionar con diente alguno por tratarse de mandíbulas edéntulas. Ver (Gráfico 2). La presencia de nervios mentonianos accesorios representó solo un $18,2 \%$ (Fig. 4).

La ubicación de los nervios mentonianos accesorios en relación al nervio mentoniano principal encontrado fue el siguiente: $66.67 \%$ de los nervios mentonianos accesorios se encuentran ubicados superior al nervio mentoniano principal, mientras que $16,67 \%$ de los nervios mentonianos accesorios se encuentran ubicados por distal e inferior al nervio mentoniano principal (Gráfico 3).

La distancia menor del borde basal al nervio mentoniano accesorio encontrado fue de $10.02 \mathrm{~mm}$ y la distancia mayor fue de $26.5 \mathrm{~mm}$. Esto nos indica una gran variabilidad en estas distancias cuya media encontrada fue de 18,12 mm y una Ds. de 6,13.

La distancia menor del reborde alveolar al nervio mentoniano accesorio fue de $8 \mathrm{~mm}$ y la distancia mayor fue de $17.58 \mathrm{~mm}$, representado en ambos casos un $16.7 \%$ del total, con una media de 13,65 y una Ds. de 3,4 lo que representa una gran variabilidad.

No se encontró la presencia de bucles del nervio mentoniano en las personas de la muestra estudiada. 
Gráfico 1. Distancia de la emergencia del nervio mentoniano y reborde alveolar

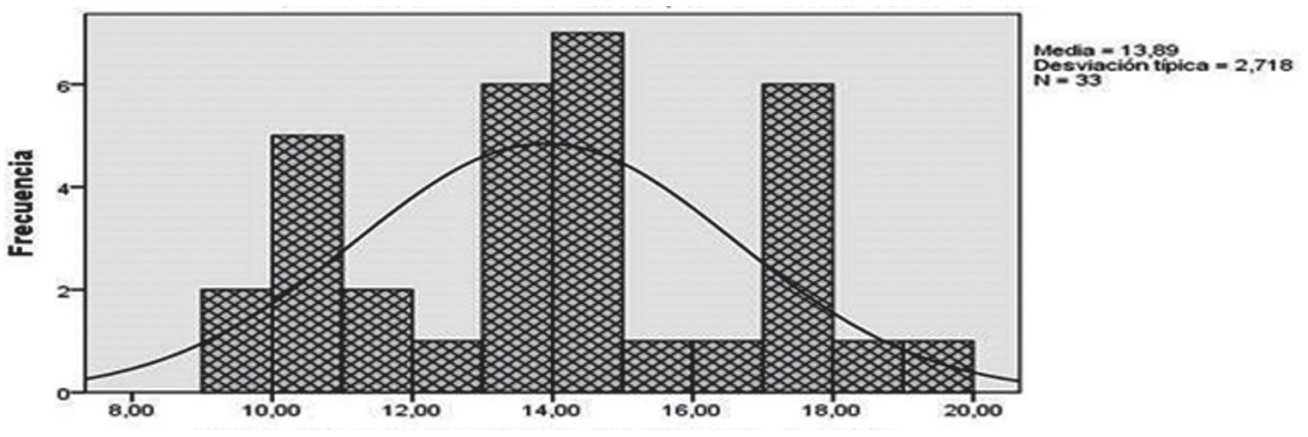

Gráfico 2 Distancia de la emergencia del nervio mentoniano y reborde alveolar

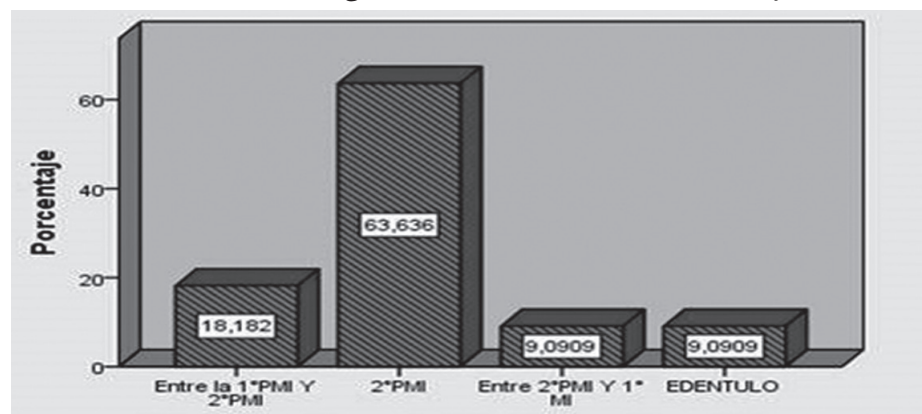

Relación de la emergencia del nervio del nervio mentoniano con los dientes de personas del Perú

\section{Gráfico 3. Ubicación de nervios mentonianos accesorios en relación} al nervio mentoniano principal

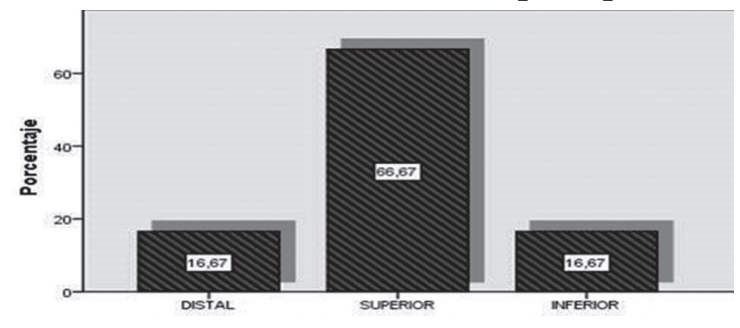

\section{Discusión}

Uno de los objetivos que se planteó para este estudio fue determinar la relación que existe entre la emergencia del nervio mentoniano y las piezas dentarias ápices, encontrándose que 63,6\% de nervios mentonianos emergen a la altura de la $2^{\text {a }}$ PMI, seguida de un $18,2 \%$ que emerge entre la $1^{\text {a }}$ PMI y la $2^{\text {a }}$ PMI, lo cual concuerda con estudios realizados por Mbajiorgu, Mawera y Asala Africa, $1998^{12}$ Greenstein y Tarnow $2006^{13}$, Ruge, Ċamargo y Ortiz $2009^{15}$ Shaik et, al. India, 2012 21 , Singh y Srivastav $2010^{9} \mathrm{Kim}$ et, al. Corea $2006^{4}$ Amorim et, al. Brasil 2008 22 , Liyian $2009^{14}$, Llayperuma, Nanayakkara y Palahepitiya Sri Lanka, 2009²3, Agarwal y Gupta Surat $2011^{20}$, Gupta y Soni India $2012^{25}$

Sin embargo también podemos ver que nuestro resultado no concuerda con estudios realizados por otros investi- gadores, como Oliveira et, al. 2009 ${ }^{16}$, Corrêa et, al. $2010^{18}$, De Almeida Filho et, al. $2011^{19}$, Haghanifar y Rokouei Iran $2009^{24}$, quienes encontraron que la emergencia del nervio mentoniano se daba mayormente entre la $1^{a}$ PMI y la 2a PMI

Como podemos apreciar la mayoría de estudios sobre la emergencia del nervio mentoniano coinciden en que un gran porcentaje de estos estaría ubicado por debajo del ápice del segundo premolar inferior, mientras que aquellos estudios que no afirman esto probablemente se deban a que la técnica de estudio se dio de manera indirecta a través de imágenes radiológicas o que fue realizado en personas con un biotipo racial diferente al nuestro. Cuando evaluamos la relación que existe entre el nervio mentoniano y el reborde alveolar, se encontró que la menor distancia era de 11 a 15,5 $\mathrm{mm}(57,6 \%)$, seguida de la mayor dis- tancia que fue 16 a $20 \mathrm{~mm}(27,3 \%)$; presentando una media de $13,89 \mathrm{~mm}$ y una Ds. de 2,72, lo que concuerda con estudios como el realizado por Agarwal y Gupta Surat $2011^{20}$ y por Shaik et, al. India, 2012 21 , donde las distancias son muy parecidas, lo cual demuestra que existe una mediana variabilidad en nuestros resultados.

Por otro lado cuando se analizó la relación entre el nervio mentoniano y el reborde mandibular, se encontró que la distancia menor fue de 11 a $15,5 \mathrm{~mm}$ $(72,7 \%)$, seguido de la distancia mayor que fue de 16 a $20 \mathrm{~mm}(21,2 \%)$, con una media de $14,69 \mathrm{~mm}$ y una Ds. de 2,72, coincidiendo con aquel estudio realizado por Kim et, al. Corea, $2006^{4}$ en donde la distancia media entre el borde superior del foramen mentoniano y la parte inferior de la mandíbula, era de $14,33 \mathrm{~mm}$ por medición directa. 
Al evaluar la presencia de nervios mentonianos accesorios en mandíbulas disecadas de cadáveres, encontramos que la presencia de éstos se dio en un 18,2\%, lo cual se asemeja a lo encontrado por Sahin, Ozkan y Gorgu Turquía, 2010 ${ }^{17}$, Singh y Srivastav $2010^{9}$ Gupta y Soni India $2012^{25}$, quienes nos dan a conocer que sí es posible encontrar nervios mentonianos accesorios en diferentes poblaciones de estudio pero que en la mayoría de los casos suele no ser muy representativo.

Otro hallazgo que no estuvo especificado dentro de nuestros objetivos, fue determinar la distancia del reborde alveolar al nervio mentoniano accesorio, encontrándose que la medida menor fue de $8.0 \mathrm{~mm}$ y la mayor de 17.58 $\mathrm{mm}$, con una media de $13,65 \mathrm{~mm}$ y una Ds. de 3,4; lo cual también representa una gran variabilidad en la distancia del agujero accesorio respecto al reborde alveolar.

Así mismo cuando evaluamos la distancia del borde basal al nervio mentoniano accesorio, encontramos que la distancia menor fue de $10.02 \mathrm{~mm}$ y la distancia mayor fue de $26.5 \mathrm{~mm}$, con una media de $18,12 \mathrm{~mm}$ y una Ds. de $6,13 \mathrm{~mm}$, lo cual también representa una gran variabilidad en estas distancias.

Si mencionamos la ubicación de los nervios mentonianos accesorios en relación al nervio mentoniano principal, esta se ubicó por encima del nervio mentoniano principal lo que representó un $66.67 \%$.

En relación a la presencia de bucles del nervio mentoniano; no se encontró en éste estudio. Sin embargo hay estudios que si manifiestan la presencia de bucles, como el de Kim et, al. 2009', Ngeow y Yuzawati Malasia 2009 ${ }^{1}$, lo cual creemos podría deberse a que nuestro estudio fue de observación directa, a diferencia de los estudios donde la técnica fue con otras ayudas, y en donde si se pudo encontrar presencia de bucles. Otra explicación sería que muchas veces los medios utilizados nos puede dar una imagen que no necesariamente se ubique clínicamente o se observe durante la disección anatómica por ser el calibre de los bucles de un grosor muy delgado.

\section{Conclusiones}

La emergencia del agujero del nervio mentoniano en relación a las piezas dentarias en cadáveres peruanos, se da mayormente por debajo de la $2^{a}$ Premolar inferior. La distancia media de la emergencia del nervio mentoniano en relación al reborde alveolar es bastante considerable. La distancia media de la emergencia del nervio mentoniano en relación al borde mandibular fue de 14,69 mm., lo cual indica que la emergencia del nervio mentoniano estaría ubicado unos milímetros más cerca al reborde alveolar que al borde mandibular. Hay presencia de nervios mentonianos accesorios en bajo porcentaje, de los cuales la mayoría se encuentran ubicados por encima del nervio mentoniano principal.

\section{Referencias bibliográficas}

1. Ngeow W, Yuzawati Y. The location of the mental foramen in a selected malay population. Journal of oral science [Internet]. 2003 [citado 22 de Julio 2013];45(3):1715. Disponible en: http://jos.dent. nihon-u.ac.jp/journal/45/3/171. pdf

2. Budhiraja V, Rastogi R, Lalwani R, Goel P, Bose SC. Study of position, shape, and size og mental foramen utilizing various parameters in dry adult human mandibles from north India. ISRN Anatomy [Internet].2013 [citado 22 de Julio de 2013]; 1-5. Disponible en: http://www.hindawi.com/journals/isrn/2013/961429/

3. Hasan T, Fausi M, Hasan D. Bilateral absence of mental foramen. International journal of anatomical variations [Internet].2010 [citado 22 de Julio de 2013];3: 167 9. Disponible en: http://www.ijav. org/2010/ijav_10_167-169.pdf

4. Kim I, et, al. Position of de mental foramen in a Korean population: a clinical and radiographic study. Implant dentistry [Internet]. 2006 [citado 22 de Julio de 2013];15(4):404-411. Disponible en: http://www.cune.nl/ index_bestanden/Kim $\% 20$ et $\% 20$ al\%202006,\%20positie\%20foramen\%20mentalis.pdf

5. Gay C, Berini L. Tratado de Cirugía Bucal Tomo I. Barcelona: Ergon; 2004. 1-40 pp.

6. Ash M, Nelson J. Anatomía Dental, Fisiología y Oclusión de Wheeler. $8^{\text {va }}$ ed. Llinas J, revisor. Madrid: Elsevier España S.A.; 2004. 383-405 pp.

7. Lopes P, Pererira G, Santos A. Location of the mental foramen in dry mandibles of adult individuals Brazil. J. Morphol. Sci.[Internet]. 2010 [citado 22 de Julio de 2013]; 27(1):23-25. Disponible en: http://jms.org.br/PDF/v27n1a07. pdf

8. Domínguez M. Jiménez O, Méndez G, López O, Oliveros G. Análisis de la posición y trayectoria del conducto alveolar inferior en tomografía volumétrica computarizada. Rev. Fac. odontolo. Univ. Antioquia [Internet]. 2010 [citado 22 de julio de 2013];22(1):12-22. Disponible en:http://aprendeenlinea.udea.edu.co/revistas/index. php/odont/article/view/5163

9. Singh R, Srivastav AK. Estudio de la posición, forma, tamaño e incidencia del foramen mentoniano y foramen mentoniano accesorio en cráneos humanos de indios adultos. International Journal of Morphology [Internet]. 2010 [citado 22 de julio de 2013];28(4): 114146. Disonible en: http://www.scielo.cl/pdf/ijmorphol/v28n4/art25. pdf

10. Serhal B, Jacobs R, Flygare L, Quirynen M, Steenberghe D. Perioperative validation of localization of the mental foramen. Dentomaxilofacial Radiology [Internet]. 2002 [citado 22 de julio de 2013];31(1):39-43. Disponible en: http://www.birpublications.org/doi/pdf/10.1038/sj/ $\mathrm{dmfr} / 4600662$

11. Granollers M, Berini L, Gay C. Variaciones de la anatomía del nervio dentario inferior Revisión bibliográfica. Anales de odontoestomatologia [Internet]. 1997 [citado 22 de julio de 2013];1:24-29. Disponible en: http://core.ac.uk/ download/pdf/16204862.pdf

12. Mbajiorgu EF, Mawera G, Asala SA, Zivanovic S. Position of the mental foramen in adult black Zimbabwean mandibles: a clinical anatomical study. The Central African journal of medicine [Internet] 1998 [citado 23 de Julio de 2013]; 44(2):24-30. Disponible en: http://europepmc.org/abstract $/ \mathrm{med} / 9675967$

13. Greenstein G, Tarnow D. The Mental Foramen and Nerve: Clinical and Anatomical Factors Related to Dental Implant Placement: A Literature Review. J Periodontol [Internet]. December 2006 [citado 23 de julio de 2013]; 77(12):1933- 
43. Disponible en: http://www. joponline.org/doi/abs/10.1902/ jop.2006.060197

14. Liyian M. Características radiográficas del foramen mentoniano en pacientes del instituto de salud oral de la FAP del 2000 al 2008. [Tesis Titulo Profesional]. [Lima-Perú]: Universidad Nacional Federico Vallareal; 2009. 12-28

15. Ruge OY, Camargo ÓA, Ortiz YP. Consideraciones anatómicas del conducto alveolar inferior. Rev Fac Odontol Univ Antioq [Internet] 2009 [citado 23 de julio de 2013]; 21(1):86-97. Disponible en: http://www.scielo.org.co/pdf/ rfoua/v21n1/v21n1a09.pdf

16. Oliveira JEM, Arújo ALD, Da Silva CM, Sousa-Rodriguez CF, Lima FJ. Morphological and morphometric study of the mental foramen on the M-CP-18 jiachenjiang point. Int. J. Morphol.[Internet]. 2009 [citado 23 de Julio de 2013];27(1):231-238. Disponible en: http://www.scielo.cl/pdf/ijmorphol/v27n1/art39.pdf

17. Sahin B, Ozkan H, Gorgu M. An Anatomical variation of mental nerve and foramen in a trauma patient. International Journal of Anatomical Variations [Internet] 2010 [citado 23 de Julio de 2013]; 3:165-6. Disponible en: http:// www.ijav.org/2010/ijav_10_165166.pdf

18. Corrêa DS, et, al. Caracterização anatômica do foramenentualem uma amostra de mandíbulas humanas secas brasileiras. Rev Bras Cir Craniomaxilofac [Internet] 2010 [citado 23 de Julio de 2013]; 13(4): 230-5. Disponible em: http://abccmf.org.br/cmf/ Revi/2010/dezembro10/07\%20 - \% 20 aracteriza $\%$ C $3 \%$ A $7 \%$ C3\%A3o\%20anat $\%$ C3\%B $4 \mathrm{mi}-$ ca $\% 20$ do $\% 20$ forame $\% 20$ mentual.pdf

19. De Almeida Filho L, et, al. Avaliação da posiçáo do forame mentual em relaçáo aos dentes e base da mandibula. Braz J Periodontol [Internet] September 2011 [citado 23 de Julio de 2013]; 21(3):91-
5. Disponible en: http://www. revistasobrape.com.br/arquivos/ set_2011/artigo14.pdf

20. Agarwal DR, Gupta SB. Morphometric analysis of mental foramen in human mandibles of South Gujarat. People's Journal of Scientific Research [Internet]. Jan 2011 [citado 23 de Julio de 2013]; 4(1)15-8. Disponible en: http://www.pjsr.org/jan-2011pdf/ Dr. \%20 Deepa\%20Rani\%20 Agarwal\%20-\%204.pdf

21. Shaik H, et, al. Morphological and morphometric study of mental foramen South Indian mandibles. Indian. J. Med. Healthcare [Internet]. June 2012 [citado 23 de Julio de 2013];1(3):64-66. Disponible en: https://scholar.google. com.pe/scholar?q=Morphologic al+and+morphometric+study+o $\mathrm{f}+$ mental+foramen+South+Indi $\mathrm{an}+$ mandibles \&btn $\mathrm{G}=\& \mathrm{hl}=\mathrm{es} \&$ as_sdt $=0 \% 2 \mathrm{C} 5 \&$ as_vis $=1$

22. Amorim M, et, al. The mental foramen in dentate and edentulous Brazilian's mandible. Int. J. Morphol [Internet].2008 [citado 23 de Julio de 2013]; 26(4):981-7. Disponible en: http:/www.scielo. cl/pdf/ijmorphol/v26n4/art33.pdf

23. Llayperuma I, Nanayakkara G, Palahepitiya N. Morphometric analysis of de mental foramen in adult Sri Lankan mandibles. Int. J. Morphol [Internet]. 2009 [citado 23 de Julio de 2013]; 27(4): 101924. Disponible en: http://www. scielo.cl/pdf/ijmorphol/v27n4/ art10.pdf

24. Haghanifar S, Rokouei M. Radiographic evaluation of the mental foramen in a selected Iranian population. Indian. J. Dent Res. [Înternet] 2009 [citado 24 de Julio de 2013]; 202):150-2. Disponible en: http://ijdr.in/article.asp?issn=0 9709290; year $=2009$; volume $=20$; is sue $=2 ;$ spage $=150$; epage $=152$; aulas $\mathrm{t}=$ Haghanifar

25. Gupta S, Soni J. Study of anatomical variations and incidence of mental foramen and accessory mental foramen in dry human mandibles. National journal of medical research [Internet]. 2012 [citado 24 de Julio de 2013];2(1): 28-30. Disponible en: http:// www.scopemed.org/fulltextpdf. php?mno= 18617

26. Velallos J.. Anatomía de la Cabeza para odontólogos. $4^{\text {ta }}$ ed. Buenos Aires: Editorial Panamericana; 2007. 54-5 pp.

27. Gómez F, Campos M. Histología, embriología e ingeniería tisular bucodental. $3^{\text {a }}$ ed. Mexico DF.: Editorial Medica Panamericana; 2009. 79-104

28. Solano E, Mendoza A, Crecimiento craneofacial y desarrollo de las arcadas dentarias. En Odontopediatria. Barcelona:Editorial Masson; 2005. 37-53. pp.

29. Ries Centeno G. Cirugía bucal: Patología, clínica y terapéutica. $9^{a}$ ed. Buenos Aires: Editorial el Ateneo; 1987. 5-9 pp.

30. Figun M, Garino R. Anatomía odontológica funcional y aplicada. $2^{\circ}$ ed. Madrid: Editorial El Ateneo; 2008. 46-50

31. Gungor K, Ozturk M, Semiz M, Brooks SL. Location of mental foramen in Turkish Population, Coll. Antropol [Internet]. 2006 [citado 24 de Julio de 2013]; 30(4):801-5. Disponible en: http://scholar.google.com.pe/scholar_url?url=http:// hrcak.srce.hr/file/43604\&hl=es \&sa $=$ X\&scisig=AAGBfm3ptAeaN99G6cg3LkSk5D_iFW-6w\&no ssl $=1 \&$ oi $=$ scholarr\&ei=FqshVfjvN sPBggSYyoK4Bg\&ved=0CBkQg AMoADAA

32. CHUMMY S. SINNATAMBY Anatomía de Last. Regional y Aplicada. $10^{\circ} \mathrm{ed}$. Barcelona: Editorial Paidotribo; 2003. 539 pags.

\section{Agradecimiento}

Al Vicerrectorado de Investigación de la Universidad Nacional Mayor de San Marcos, por el apoyo financiero, operativo, de consultoría y administrativo que permitió desarrollar nuestro estudio.

\section{Conflicto de intereses}

Los autores declaramos no tener conflictos de interés en éste trabajo. 